\title{
Interactive comment on "An IBBCEAS system for atmospheric measurements of glyoxal and methylglyoxal in the presence of high $\mathrm{NO}_{2}$ concentrations" by Jingwei Liu et al.
}

\section{Anonymous Referee \#1}

Received and published: 3 April 2019

Liu et al. describe a new incoherent broadband cavity enhanced absorption spectrometer (IBBCEAS) for atmospheric measurements of NO2, glyoxal, and methyl glyoxal. The instrument has been thoroughly characterized and performs well compared to existing methods. As a new feature, the instrument used a photolytic converter (NPC) to selectively reduce the amount of $\mathrm{NO} 2$ in their sample stream.

Major revisions will be required before this paper is acceptable for publication.

(1) There are too many figures in the current draft. Many are not necessary.

(2) Some figures are unclear as the captions are too short. 
(3) I was not convinced of the merits of the NPC. Is the NPC even necessary? It seems that (valid) data could be obtained without it, though at a slightly higher noise level.

(4) Does the NPC generate glyoxal and methyl glyoxal when ambient air containing organic compounds is sampled? The MCM lists 127 precursors for glyoxal and 143 precursors for methyl glyoxal. I havn't gone through these lists in detail, but it seems that more characterization experiments are warranted in my opinion. It seems to me that the NPC may generate more issues than it solves.

(5) The convolved spectrum of MGLY (Figure 4, thick purple line) does not look correct.

Specific comments

pg 2, line 28 "Thalman et al. first encountered the challenge of fitting GLY and MGLY absorption spectra in the presence of high NO2 concentrations (Thalman et al., 2015). To our knowledge, an effective method has not yet to solve this problem." This is a bit of mischaracterization - Thalman et al. [2015] stated that "For both CE-DOAS and BBCEAS ... we do not find significant bias; i.e., an upper-limit change in glyoxal due to $\mathrm{NO} 2$ is derived as +/-200 pptv glyoxal in the presence of 200 ppbv NO2 (or bias of 1 pptv glyoxal/1 ppbv NO2)."- see also major comment \#3.

pg 7 line 31 . Consider adding a digit to each uncertainty as otherwise $4 \%+2 \%=4 \%$.

pg 9 line 23. "The presence of high NO2 concentrations may affect the spectral fitting of GLY and MGLY". Perhaps, but this is not obvious from Figure 9 (which seem OK to me). To make their point, it seems like the authors could perform a rather easy experiment: Deliver a constant concentration of GLY (or MGLY) and add increasing amounts of NO2 to this blend. Are the correct GLY (or MGLY) concentrations retrieved?

Figure 1 (a) Consider adding dimensions to this sketch, such as the inter-mirror distance

Figure 2 is not necessary in my opinion. Simply state the result in the text. 
Figure 3. You may want to state the dimensions of the mixing chamber, temperature of the cold trap, and flow rates.

Figure 4 The convolved methylglyoxal spectrum does not look right.

Figure 6. This factor will be flow rate dependent, and the sample flow rate should be stated in the caption. Please zoom in on the ratio more (0.85 to 0.95$)$ and state the standard deviation of this measurement. The effective path was determined from a comparison of measured to known concentration; the figure is misleading as it is suggested that it was determined by turning the purge gases on and off (filling the purge flow volumes would take much more time than shown here). Consider clarifying this in the text.

Figure 11 The figure caption should describe the experiment conducted here in more detail. Figure 11 (c) The figure caption should indicate what is meant by the yellow overlay. Are the times displayed correct? It seems that the NO2 mixing ratios stay at the high levels well after the NPC was turned on, but increase again before the NPC is turned off?

Figure 12 - what does 'fixing' the concentration mean? What is 'first', 'second', 'third', and 'fourth'?

Figure 13 (b). There are two sets of concentrations displayed - which one is methylglyoxal, and what is the other one?

Figure 14. The figure caption should indicate what is meant by the yellow overlay. (b) Are the times displayed correct? It seems that the NO2 mixing ratios stay at the high levels well after the NPC was turned on, but increase again before the NPC is turned off? The photo-oxidation of certain organic molecules (e.g., benzene, toluene etc.) can yield glyoxal or methylglyoxal. It looks like this is happening here. Consider evaluating the NPC while sampling an atmospheric background of those VOCs.

Printer-friendly version

Fig 14 (c) The caption does not adequately describe what is shown in the figure.

Discussion paper 
Interactive comment on Atmos. Meas. Tech. Discuss., doi:10.5194/amt-2019-40, 2019.

AMTD

Interactive comment 